\title{
Evaluation of the Effects of a Moderate Intensity Static Magnetic Field Application on Human Osteoblast-Like Cells
}

\author{
Carla Cunha $^{1,2}$, Silvia Panse ri ${ }^{1,2, *}$, Maurilio Marcacci ${ }^{1}$, Anna Tampieri ${ }^{2}$ \\ ${ }^{1}$ Laboratory of Biomechanics and Technology Innovation, Rizzoli Orthop aedic Institute, Bologna, 40136, Italy \\ ${ }^{2}$ Laboratory of Bioceramics and Bio-hybrid Composites, Institute of Science and Technology for Ceramics, National Research Council, \\ Faenza, 48018, Italy
}

\begin{abstract}
There is a general interest in the effects of magnetic fields on human tissue, for biomedical imaging, cancer therapy or tissue engineering applications. In particular the orthopaedic field may greatly benefit from magnetic scaffolds, magnetic fixation and magnetic delivery techniques. In this study, MG-63 osteoblast-like cells were analysed in vitro after exposure to a $320 \mathrm{mT}$ static magnetic field (SMF), either continuously or applied for $1 \mathrm{~h}$ at any 24-hour interval. Results demonstrate that SMF causes a reduction in cell number after 7 days of exposure, as demonstrated by the MTT assay. This reduction in proliferation is not associated to an increase in Lactate Dehydrogenase production, a marker of cellu lar stress and/or disruption of memb rane integrity. Osteocalcin secretion increased at day 3 for the condition $1 \mathrm{~h} /$ day exposure and this effect was reversed after 7 days. Instead, the continuous application of a SMF resulted in a significantly decreased osteocalcin release at day 7. Cell distribution, morphology and cytoskeleton organization were unaltered, with the typical osteoblastic morphology maintained and normal distribution of cytoplasmic actin fibrils, as demonstrated by phalloidin staining. Gene expression analysis of COL1A1, A LPL and RUNX2 show no alterations respect to control. These results suggest that such a moderate intensity static magnetic field has a detrimental effect on cell proliferation and osteocalcin secretion, while maintaining morphological features and gene expression unaltered. The in vitro effects of magnetic fields depend on cell type, magnetic field intensity and modality of application. This study gives a contribution to understand moderate strength static magnetic field effects on human tissue, with particular importance for the orthopaedic field.
\end{abstract}

Keywo rds Static Magnetic Field, Osteoblast Behaviour, Gene Expression, Magnetic Scaffo lds

\section{Introduction}

There has been an increasing interest in the study of magnetic field employment in the clinical setting, either for magnetic resonance imaging, for magnetically guided delivery of biomolecules for tissue engineering applications or cancer therapy. In particular in the orthopaedic field, the use of static magnetic fields finds a broad range of applications, from the ones mentioned to the possibility of having in situ magnetic fixed stations for prosthesis or scaffold fixation. In fact, our laboratory has recently developed a simulated scaffold fixation technique for osteochondral defect surgery that makes use of magnetic forces attracting the scaffold towards the implanted position ${ }^{1}$.

Also the use of superparamagnetic nanoparticles (MNPs)

* Corresponding author:

s.panseri@biomec.ior.it (Silvia Panseri)

Published online at http:/journal.sapub.org/ajbe

Copyright (C) 2012 Scientific \& Academic Publishing. All Rights Reserved for biological and medical purposes has been increasing, either as contrast agent for diagnostic MRI imaging, for its hyperthermia effect, for magnetic drug delivery and for tissue engineering applications ${ }^{2-6}$. We have recently demonstrated that intrinsically magnetic iron-substituted hydroxyapatite nanoparticles significantly increase osteoblast proliferation and activity when exposed to a static magnetic field ${ }^{7}$ and the same nanoparticles have been used together with a biodegradable poly(caprolactone) core for the development of a magnetic scaffold for bone tissue engineering ${ }^{8}$. Also, we have introduced magnetite into the structure of a macroporous hydroxyapatite ceramic scaffold, which demonstrated in vitro biocompatibility, either in the presence or absence of a magnetic field, and good in vivo osteointegration 9. Moreover, we have designed two hydroxyapatite/collagen (70/30 wt \%) magnetic scaffolds, magnetized with two different techniques: direct nucleation of biomimetic phase and MNPs on self-assembling collagen fibers and scaffold impregnation in a ferro-fluid solution ${ }^{10,11}$. Recently, we have designed magnetic biohybrid scaffolds made of collagen and intrinsically magnetic iron-substituted 
hydroxyapatite for bone regeneration ${ }^{12}$. The possibility of using superparamagnetic phases mostly relies on the application of an external magnetic field able to activate such materials and/or to drive bio mo lecule loaded MPN to desired specific sites. The effects of such magnetic fields on the human tissue need to be well investigated in order to understand the possible range of application of such magnetic devices and its implication for the different biomedical applications. Cell culture studies directed at exploring both the biocompatibility of magnetic devices and also the effects of magnetic fields in biological tissues are needed. In this context, this study analysed for the first time the effect of a moderate intensity static magnetic field ( 320 $\mathrm{mT}$ ) on MG-63 human osteoblast cell culture, and intends to give a contribute to deepen the knowledge on possible applications of magnetic devices driven by magnetic forces in the clinical setting.

\section{Materials and Methods}

\subsection{In Vitro Cell Cul ture}

MG-63 human osteoblast-like cells (Lonza, Italy) were cultured in DMEM/F12 medium (PAA, Austria) containing $10 \%$ FBS and Penicillin-Streptomyc in $(100 \mathrm{U} / \mathrm{ml}-100 \mu \mathrm{g} / \mathrm{ml})$ and kept at $37^{\circ} \mathrm{C}$ in an atmosphere of $5 \% \mathrm{CO}_{2}$. Cells were detached from culture flasks by trypsinization, centrifuged and resuspended. Cell number and viability were assessed with the trypan-blue dye exclusion test. $2.5 \times 104$ cells per well were plated into a 24-mu ltiwell and cultured for 1, 3 and 7 days, in the absence (control) or presence of an external magnet, which relied on the application of a commercially available static magnetic field of $320 \mathrm{mT}$ under the multiwell plates (MagnetoFACTOR-24, Chemicell, Germany), which ensures equal application of the magnetic field under each well. A third condition relied on the exposure of cells to the static magnetic field for 1 hour at any 24-hour interval.

All cell handling procedures were performed in a sterile laminar flow hood. Cell culture incubation was performed at $37^{\circ} \mathrm{C}$ with $5 \% \mathrm{CO}_{2}$.

\subsection{MTT Assay}

The MTT reagent (3-(4,5-dimethylthiazol-2-yl)-2,5diphenyltetrazoliu mb ro mide) (Invitrogen) was prepared at 5 $\mathrm{mg} / \mathrm{ml}$ in $1 \mathrm{x}$ PBS. Cells were incubated with the MTT reagent $1: 10$ for $2 \mathrm{~h}$ at $37^{\circ} \mathrm{C}$. Medium was discarded and cells incubated with $1 \mathrm{ml}$ of Dimethyl sulfoxide (Sig ma) for 15 $\mathrm{min}$. In this assay, the metabolically active cells react with the tetrazolium salt in the MTT reagent to produce a formazan dye that can be observed at $\lambda \max$ of $570 \mathrm{~nm}$, using a Multiskan FC Mic roplate Photometer (Thermo Scientific). This absorbance is directly proportional to the number of metabolically active cells. Mean values of absorbance were determined. Three samples were analysed per group per time point.

\subsection{Lactate Dehydrogenase Assay}

Liqui Lactate Dehydrogenase (LDH) assay (Futura System) was performed according to manufacturer's instructions. Brie fly, LDH solution was prepared by mixing 4 parts of Buffer solution to 1 part of Substrate solution. 20 $\mu 1$ of cell medium was added to $1 \mathrm{ml}$ of LDH solution. Absorbance was read at $0,1,2$ and $3 \mathrm{~min}$ at $\lambda \max$ of $340 \mathrm{~nm}$, using a Multiskan FC Microplate Photometer (Thermo Scientific). LDH activity was determined by calculating the $\Delta \mathrm{OD} / \mathrm{min}$. LDH activity was expressed as U/l. Three samples were analysed per group per time point.

\subsection{Human Osteocalcin Elisa}

Osteocalcin (OST) secretion was measured by the Human Osteocalcin ELISA kit (Invitrogen), following manufacturer's instructions. Briefly, $25 \mu 1$ of cell medium was incubated with $100 \mu 1$ of anti-OST-HRP antibody for 2 hours at room temperature, within the supplied microtiter plate, coated with a second anti-OST antibody. After washing, $100 \mu \mathrm{l}$ of chromogenic solution was added to the wells and plate incubated at room temperature in the dark. Reaction was stopped 25 min after addition of $100 \mu 1$ of stop solution. Absorbance was read at $\lambda \max$ of $450 \mathrm{~nm}$, using a Multiskan FC Microplate Photometer (Thermo Scientific). The amount of osteocalcin was determined by interpolation from a standard curve of known concentrations. Osteocalcin concentration was expressed as $\mathrm{ng} / \mathrm{ml}$. Three samples were analysed per group per time point.

\subsection{Phalloi din Immunofluorescence Staining}

Cells were washed with $1 \times$ PBS for 5 min, fixed with $4 \%$ $(\mathrm{w} / \mathrm{v})$ paraformaldehyde for $15 \mathrm{~min}$ and washed with $1 \times$ PBS for $5 \mathrm{~min}$. Permeabilization was performed with $1 \mathrm{x}$ PBS with $0.1 \%(\mathrm{v} / \mathrm{v})$ Triton $\mathrm{X}-100$ for $5 \mathrm{~min}$. FITC-conjugated Phallo idin antibody (Invitrogen) 1:500 in 1x PBS was added for $20 \mathrm{~min}$ at room temperature in the dark. Cells were washed with 1x PBS for 5 min and incubated with $300 \mathrm{nM}$ DAPI (4'-6-Diamid ino-2-phenylindole) solution (Invitrogen) in 1x PBS for $5 \mathrm{~min}$. Images were acquired by an Inverted Ti-E fluorescence microscope (Nikon). Two samples per group were analysed.

\subsection{Total RNA Extraction and Reverse Transcription}

At each time point, cells were collected by trypsinization, centrifuged, resuspended in RNAlater solution and kept at $-20^{\circ} \mathrm{C}$. Total RNA isolation was performed by use of the Tris reagent, followed by the Purelink RNA Mini kit according to manufacturer's instructions. In particular, we have used a on-column DNAse treatment by use of the Pure link DNAse set. Purified total RNA was eluted with THE RNA storage solution and kept at $-80^{\circ} \mathrm{C}$ until reverse transcription. RNA integrity was analysed by native agarose gel electrophoresis (1.5\% agarose in $1 \times$ TBE, with $1 \mu \mathrm{g} / \mathrm{ml}$ of ethid iu m bro mide), run in $1 \mathrm{x}$ TBE buffer and quantification performed by the Qubit ${ }^{\circledR}$ 2.0 Fluorometer (Invitrogen), together with the Qubit ${ }^{\circledR}$ RNA BR assay kit, following manufacturer's instructions. cDNA was obtained by using the High capacity 
cDNA reverse transcription kit, accord ing to manufacturer's instructions. In particular, we have used $1.5 \mu \mathrm{g}$ of total RNA in a $20 \mu \mathrm{l}$ final reaction volume for each sample, as follows: $2 \mu 1$ of $10 x$ RT buffer, $0.8 \mu 1$ of $25 x$ dNTP mix, $2 \mu 1$ of $10 x$ RT primers, $1 \mu 1$ of RNAse inhibitor, $3.2 \mu$ of Nuclease free water plus $10 \mu \mathrm{l}$ of temp late. Reaction conditions: $25^{\circ} \mathrm{C}$ for $10 \mathrm{~min}, 37^{\circ} \mathrm{C}$ for $120 \mathrm{~min}, 85^{\circ} \mathrm{C}$ for $5 \mathrm{~min}$. cDNA was kept at $-20^{\circ} \mathrm{C}$ until qPCR. All reagents and instruments were from Life Technologies.

\subsection{Quantitative Real-time Polymerase Chain Reaction (qPCR)}

Quantification of Collagen, type I, alpha 1 (COL1A1), Alkaline Phosphatase (ALPL), Runt-related transcription factor 2 (RUNX2) and glyceraldehyde 3-phosphate dehydrogenase (GAPDH) gene expression was performed by use of the StepOne ${ }^{\text {TM }}$ Real-Time PCR System (Applied Biosystems). We used the Taqman gene expression mastermix and the following Taqman gene expression as says: GA PDH: Hs99999905_m1, COL1A 1: Hs 00164004 _m1, ALPL: Hs 01029144_m1 and RUNX2: Hs00231692_ $\mathrm{m} 1$, all with the Fam ${ }^{\mathrm{TM}}$ dye as reporter. $1 \mu \mathrm{l}$ of $\mathrm{cDNA}$ was used in a $10 \mu \mathrm{l}$ final reaction volume, as follows: $5 \mu 1$ of $2 \mathrm{x}$ mastermix, $0.5 \mu 1$ of $20 x$ gene expression assay, $3.5 \mu l$ of Nuclease free water plus $1 \mu$ l of template. qPCR reaction was performed on a 48-well plate adhesive sealed, as follows: 40 cycles at $95^{\circ} \mathrm{C}$ for $15 \mathrm{sec}, 60^{\circ} \mathrm{C}$ for $1 \mathrm{~min}$, after an initial denaturation step of $50^{\circ} \mathrm{C}$ for $2 \mathrm{~min}$ and $95^{\circ} \mathrm{C}$ for $10 \mathrm{~min}$. Data was collected using the OneStep Software (Applied Biosystems ) and relative quantification was performed using the comparative threshold $(\mathrm{Ct})$ method $(\Delta \Delta \mathrm{Ct})$, where relative gene expression level equals $2-\Delta \Delta \mathrm{Ct}$ (Livak and Schmittgen, 2001), as described by the manufacturer. In detail, changes in gene expression level of the target genes COL1A1, ALPL and RUNX2 were calculated by norma lization to the reference GAPDH and by normalization to the control condition, used as calibrator, within each sample set. Changes in gene expression were reported as fold changes of target re lative to controls and represent averages from triplicate measurements. All reagents and instruments used for gene expression analysis were from Life Technologies.

A

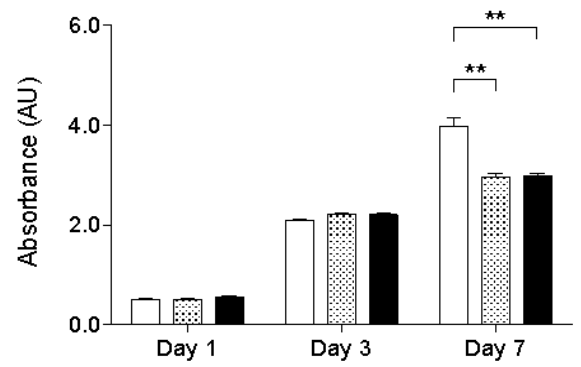

B

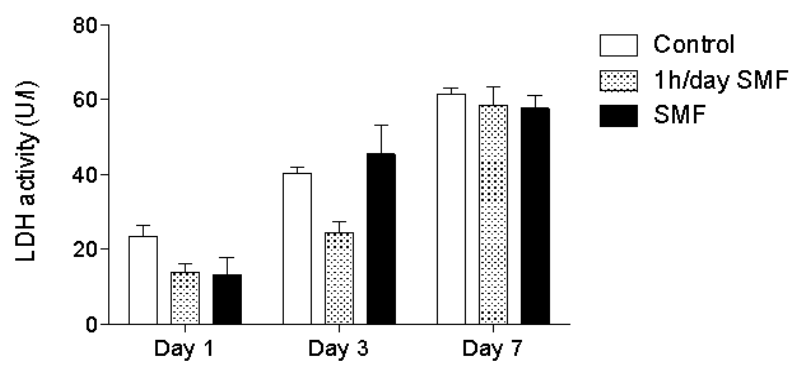

Figure 1. Analysis of cell proliferation and cellular stress. Quantification of cell proliferation was performed by the MTT assay (A) and quantification of cellular stress was performed by the Lactate Dehydrogenase (LDH) assay (B) at 1,3 and 7 days for control osteoblasts, osteoblasts exposed for $1 \mathrm{~h} /$ day to a static magnet ic field (SMF) or continuously exposed to a SMF. $n=3, * * \mathrm{p} \leq 0.01$

\subsection{Statistical Analysis}

For MTT, LDH and Osteocalcin experiments, results were expressed as MEAN \pm SEM plotted on graph $(n=3)$. Analys is of differences between groups was made by one-way ANOVA for each time point, followed by Dunnett's post-hoc test. For gene expression experiments, results were expressed as RQ with MAX and MIN values plotted on graph $(n=3)$. All statistical analysis made use of the GraphPad Prism software (version 5.0), with statistical significance set at $\mathrm{p} \leq 0.05$.

\section{Results and Discussion}

In this study, we analysed in vitro the effect on a MG-63 osteoblast culture of a moderate strength static magnetic field (SMF) of $320 \mathrm{mT}$, a value that is within the range (1 mT to $1 \mathrm{~T})$ demonstrated to have particular implications in a number of biological phenomena ${ }^{13,14}$. We have used two different conditions: continuous exposure and exposure for 1h per day, envisaging a possible clinical setting application, where the patient would be submitted to therapy for a restricted period of time. Experimental time points were for all experiments 1, 3 and 7 days, except for gene expression analys is where only day 7 was considered.

\subsection{Application of a Static Magnetic Field Reduces Cell Proliferation}

The MTT assay is a reliable method to quantify cell proliferation at each time point. Results demonstrate that when exposed for either $1 \mathrm{~h} /$ day or continuously to a SMF, cells continue to grow from day 1 to day 7 (Fig. 1A), but presenting a considerably reduced number of cells respect to control, at day $7 \quad(\mathrm{p} \leq 0.01)$. Still, this reduction in proliferation observed at day 7 is not associated to an increase in cellular apoptosis, stress or disruption of membrane integrity, in fact quantification of the cellular stress marker Lactate Dehydrogenase (LDH), released when cell membrane integrity is disrupted, showed no difference between groups at any of the time points (Fig. 1B). 
The observed reduction in proliferation is in contrast to a previous study that used murine MC3T3-E1 osteoblasts and showed that a moderate SMF resulted in enhanced proliferation ${ }^{13}$. Still, this was a different cell line and the intensity of the SMF was less than half $(150 \mathrm{mT})$ the one used in this study $(320 \mathrm{mT})$, which could explain different results.

Another study instead demonstrated MG-63 osteoblasts increased proliferation when exposed to a $400 \mathrm{mT}$ SMF, yet this study was performed on a poly-L-lactide substrate ${ }^{15}$.

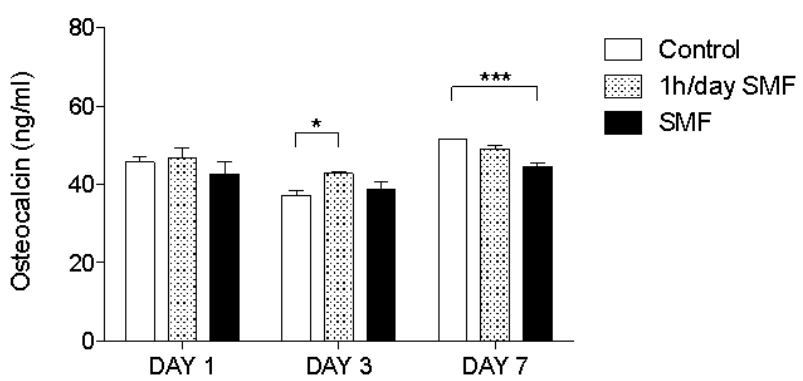

Figure 2. Analysisof the osteogenic marker ost eocalcin. Quantification of released osteocalcin was performed by ELISA at 1,3 and 7 days for control osteoblasts, osteoblasts exposed for $1 \mathrm{~h} /$ day to a stat ic magnet ic field (SMF) or continuously exposed to a SMF (n=3). * $\mathrm{p} \leq 0.05, * * * \mathrm{p} \leq 0.001$

\subsection{Osteocalcin is Reduced after 7 Days of Expos ure}

Osteocalcin is secreted by osteoblasts and is the major non collagenous component of the bone extracellular matrix, being used as a reliable marker for bone formation. The quantification of the osteocalcin released by MG-63 osteoblasts to the cell culture was performed by the Human Osteocalcin Elisa Kit. Results demonstrate that application of a SMF for 1h/day increases osteocalcin release at day 3, respect to control $(\mathrm{p} \leq 0.05)$, while this effect is reversed after 7 days (Fig. 2). Instead, the continuous application of a SMF resulted in decreased osteocalcin release, respect to control $(p \leq 0.001)$. These results might indicate a beneficial effect of the application of a SMF for only $1 \mathrm{~h} /$ day at the short time, which may result prejudicial if applied continuously and for a more extended period of time. Another study has demonstrated that application of a $150 \mathrm{mT}$ SMF on murine MC3T3-E1 osteoblasts induced increased osteocalcin secretion after 15 days ${ }^{13}$. Overall, such results suggest important differences between cell types, magnetic field intensity and duration and mode of application, so that individual conditions must be optimized for specific clinical application set-ups.

\subsection{No differences in Cellul ar Distribution or Mor phology}

In order to determine effects of the SMF on cellular distribution, morphology and cytoskeleton organization, at day 7 , we have performed immunofluorescence analys is against phalloidin, a to xin that binds F-actin filaments, and DAPI, a nucleic acid stain commonly used as cell nuclei marker. Overall, we have not observed any differences between osteoblasts cultured as control or exposed to the SMF. Cells were nicely distributed throughout the cell culture well (Fig. 3A,B), connections between the cells remained unaltered and the typical osteoblastic morphology was not lost (Fig. 3C,D). The distribution of the actin fibrils on the cell cytoplasm was normal (Fig. 4). A previous study instead has shown that MG-63 cells treated with $400 \mathrm{mT}$ SMF had more differentiated morphologic features already after 3 days ${ }^{16}$, while a SMF of a mere $6 \mathrm{mT}$ has been shown to influence cell membrane integrity ${ }^{17}$. Our morphological results instead are coherent with the $\mathrm{LDH}$ assay results, which measure membrane disruption, in showing an absence of SMF effects on cell membrane integrity.
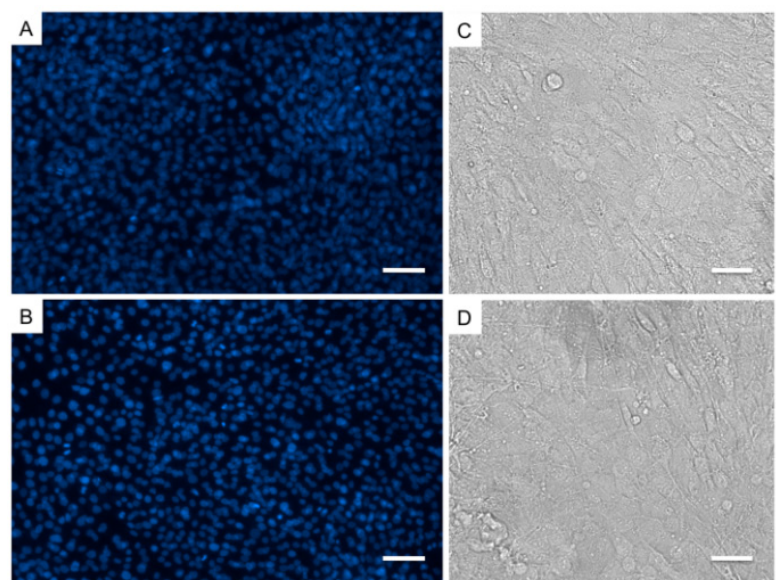

Figure 3. Analysis of cell distribution and morphology. MG-63 osteoblast-like cell culture under control conditions $(\mathrm{A}, \mathrm{C})$ and continuously exposed to a static magnetic field (B,D), at day 7. DAPI staining in blue $(A, B)$ shows no difference in cellular distribution and nuclei int egrity. High magnification brightfield imaging (C,D) shows typical osteoblast morphology and intercellular connections. Scale bars: A,B) $100 \mu \mathrm{m}, \mathrm{C}, \mathrm{D})$ $50 \mu \mathrm{m}$
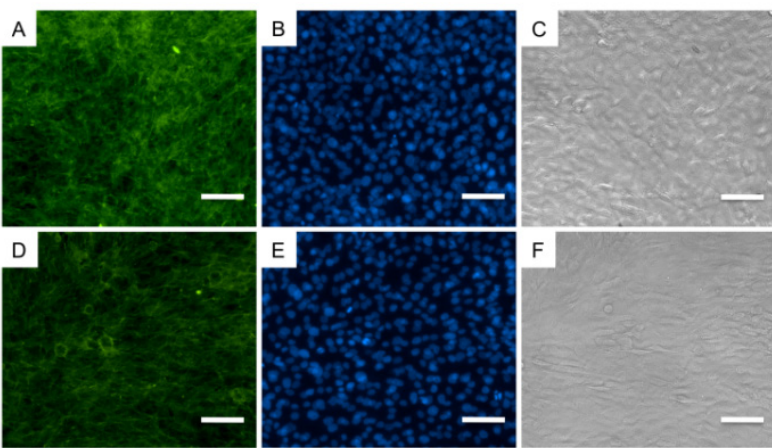

Figure 4. Act in immunofluorescence analysis. Detailed analysis of cytoplasm matrix organization was performed at day 7 by immunofluorescence staining against phalloidin, a marker of actin filaments (A,D, in green), and respective DAPI staining (B,E, in blue) and brightfield imaging $(\mathrm{C}, \mathrm{F})$. A,B,C refer to the control condition, $\mathrm{D}, \mathrm{E}, \mathrm{F}$ refer to continuous exposure to a static magnetic field. Scale bars: $100 \mu \mathrm{m}$ 

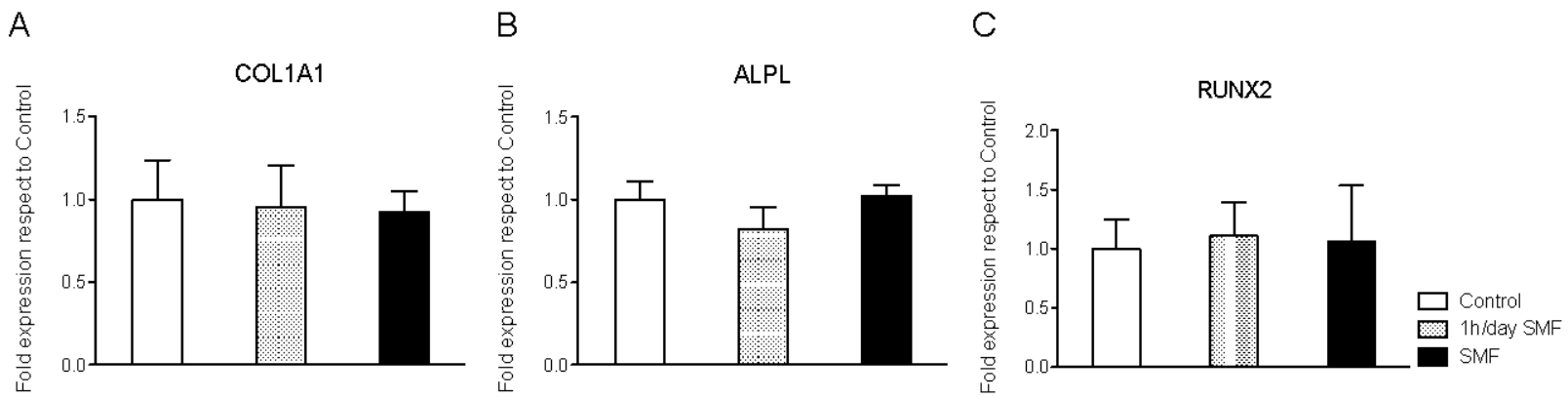

Figure 5. Gene expression analysis. Relative quantification of gene expression after 7 days of MG-63 culture under the application of a cont inuous static magnet ic field (SMF) or under the application of a SMF for $1 \mathrm{~h}$ per day, respect to the control, aft er normalization to the GAPDH reference. A) Collagen type I, alpha 1 (COL1 A1), B) Alkaline Phosphatase (ALPL), C) Runt-related transcription factor 2 (RUNX2)

\subsection{No Alteration in COL1 A1, ALPL or RUNX2 Gene Expression}

Gene expression analysis was performed by reverse transcription quantitative real time PCR, at day 7 , for groups control, SMF and 1h/day SMF. Collagen type I, alpha 1 (COL1A1), alkaline phosphatase (ALPL) and Runt-related transcription factor 2 (RUNX2) were analysed, using GAPDH as reference. Collagen type $I$ is the main extracellular matrix component of osteoblast cultures, alkaline phosphatase is a marker of osteoblast activity and Runx2 is associated with osteoblastic differentiation. No difference was observed in their relative gene expression for any of these proteins. Instead, a previous study demons trated that a low intensity SMF has resulted in decreased ALPL and COL1A 1 gene expression on osteoblast cell cultures for up to 14 days ${ }^{18}$.

\section{Conclusions}

This study analysed the effects of a static magnetic field intensity of $320 \mathrm{mT}$ on MG-63 hu man osteoblast-like cells in vitro. Our results suggest a detrimental effect on cell proliferation by the application of a SMF, either continuously or applied for $1 \mathrm{~h} /$ day. This reduction was not associated to an increase in cellular apoptosis, stress or disruption of membrane integrity, quantified by the cellular stress marker Lactate Dehydrogenase. Quantification of osteocalcin release, a marker for bone formation, demonstrated that application of a SMF for $1 \mathrm{~h} /$ day increases osteocalcin release at day 3 , while this effect was reversed after 7 days. Instead, the continuous SMF application resulted in decreased osteocalcin release, which might indicate a beneficial effect of the application of a SMF for only $1 \mathrm{~h} /$ day at the short time, which may result prejudicial if applied continuously and for a more extended period of time. No diffe rences were seen by immunofluorescence analys is in cellular distribution, morphology or cytosk elet on organization after 7 days in culture. Quantitative PCR analysis for COL1A 1, ALPL and RUNX2, all markers of osteoblast activity, identified no alterations in gene expression introduced by the SMF to the cell culture. For sure the effects of magnetic fields on in vitro cell culture depend on cell type, magnetic field intensity and duration and mode of application. This study gives a contribution to understand moderate strength static magnetic field effects on human tissue.

\section{ACKNOWLEDGEMENTS}

The authors acknowledge the financial support from European Union project MAGISTER NMP3-LA-2008214685 .

\section{REFERENCES}

[1] Russo A, Shelyakova T, Casino D, Lopomo N, Strazzari A, Ortolani A, Visani A, Dediu V, Marcacci M. A new approach to scaffold fixation by magnetic forces: Application to large osteochondral defects. Med Eng Phys 2012.

[2] Amirfazli A. Nanomedicine: magnetic nanoparticles hit the target. Nat Nanotechnol 2007;2(8):467-8.

[3] Arruebo M, Fernandez Pacheco R, Ibarra MR, Santamaria J. Magnetic Nanoparticles for Drug Delivery. Nano Today 2007;2:22-32.

[4] Foy SP, Manthe RL, Foy ST, Dimitrijevic S, Krishnamurthy $\mathrm{N}$, Labhasetwar V. Optical imaging and magnetic field targeting of magnetic nanoparticles in tumors. ACS Nano 2010;4(9):5217-24.

[5] Jain TK, Reddy MK, Morales MA, Leslie-Pelecky DL, Labhasetwar V. Biodistribution, clearance, and biocompatibility of iron oxide magnetic nanoparticles in rats. Mol Pharm 2008;5(2):316-27.

[6] Prijic S, Scancar J, Romih R, Cemazar M, Bregar VB, Znidarsic A, Sersa G. Increased cellular uptake of biocompatible superparamagnetic iron oxide nanoparticles into malignant cells by an external magnetic field. J Membr Biol 2010;236(1):167-79.

[7] Panseri S, Cunha C, D'Alessandro T, Sandri M, Giavaresi G, Marcacci M, Hung CT, Tampieri A. Intrinsically superparamagnetic Fe-hydroxyapatite nanoparticles positively influence osteoblast-like cell behaviour. J Nanobiotechnology 2012;10(1):32. 
[8] Banobre-Lopez M, Pineiro-Redondo Y, De Santis R, Gloria A, Ambrosio L, Tampieri A, Dediu V, Rivas J. Poly (caprolactone) based magnetic scaffolds for bone tissue engineering. Journal of Applied Physics 2011;109(7).

[9] Panseri S, Cunha C, D'Alessandro T, Sandri M, Russo A, Giavaresi G, Marcacci M, Hung CT, Tampieri A. Magnetic hydroxyapatite bone substitutes to enhance tissue regeneration: evaluation in vitro using osteoblast-like cells and in vivo in a bone defect. PLoS One 2012;7(6):e38710.

[10] Bock N, Riminucci A, Dionigi C, Russo A, Tampieri A, Landi E, Goranov VA, Marcacci M, Dediu V. A novel route in bone tissue engineering: magnetic biomimetic scaffolds. Acta Biomater 2010;6(3):786-96.

[11] Panseri S, Russo A, Giavaresi G, Sartori M, Veronesi F, Fini M, Salter DM, Ortolani A, Strazzari A, Visani A and others. Innovative magnetic scaffolds for orthopedic tissue engineering. J Biomed Mater Res A 2012.

[12] Tampieri A, Landi E, Valentini F, Sandri M, D'Alessandro T, Dediu V, Marcacci M. A conceptually new type of bio-hybrid scaffold for bone regeneration. Nanotechnology 2011;22(1):015104.

[13] Ba X, Hadjiargy rou M, DiMasi E, Meng Y, Simon M, Tan Z, Rafailovich MH. The role of moderate static magnetic fields on biomineralization of osteoblasts on sulfonated polystyrene films. Biomaterials 2011;32(31):7831-8.

[14] Wang Z, Che PL, Du J, Ha B, Yarema KJ. Static magnetic field exposure reproduces cellular effects of the Parkinson's disease drug candidate ZM241385. PLoS One 2010;5(11): e13883.

[15] Feng SW, Lo YJ, Chang WJ, Lin CT, Lee SY, Abiko Y, Huang HM. Static magnetic field exposure promotes differentiation of osteoblastic cells grown on the surface of a poly-L-lactide substrate. Med Biol Eng Comput 2010; 48(8): 793-8.

[16] Huang HM, Lee SY, Yao WC, Lin CT, Yeh CY. Static magnetic fields up-regulate osteoblast maturity by affecting local differentiation factors. Clin Orthop Relat Res 2006;447: 201-8.

[17] Dini L, Abbro L. Bioeffects of moderate-intensity static magnetic fields on cell cultures. Micron 2005;36(3):195-217.

[18] Denaro V, Cittadini A, Barnaba SA, Ruzzini L, Denaro L, Rettino A, De Paola B, Papapietro N, Sgambato A. Static electromagnetic fields generated by corrosion currents inhibit human osteoblast differentiation. Spine (Phila $\mathrm{Pa}$ 1976) 2008;33(9):955-9. 